\title{
International workshop on eigenvalue problems: algorithms; software and applications, in petascale computing (EPASA2018)
}

\author{
Tetsuya Sakurai $^{1} \cdot$ Toshiyuki Imamura $^{2} \cdot$ Kengo Nakajima $^{3}$
}

Published online: 12 June 2019

(c) The JJIAM Publishing Committee and Springer Japan KK, part of Springer Nature 2019

The next eight articles of the present issue are from some of the speakers who presented them as papers at the "International workshop on eigenvalue problems: algorithms; software and applications, in petascale computing (EPASA2018)". The workshop was held at the Tsukuba International Congress Center EPOCHAL TSUKUBA, Tsukuba, Ibaraki, Japan, March 5-6, 2018. More than 40 participants including eight invited speakers from China, France, Japan, Spain, and the United States presented talks, exchanged ideas, and had active discussions on a variety of topics on eigensolvers, theoretical analysis, software, and their applications.

EPASA started with the support of the JST CREST project "development of an eigen-supercomputing engine using a post-petascale hierarchical model". The EPASA series has created an international community of researchers studying eigenvalue problems.

The guest editors are grateful for the invitation to the Japan Journal of Industrial and Applied Mathematics extended by the late Professor Masaaki Sugihara and would like to express their sympathy to his family. They also thank all the authors for their contributions and the reviewers for their efforts.

March 2019

Guest editors:

Tetsuya Sakurai

Toshiyuki Imamura

Kengo Nakajima

\footnotetext{
Tetsuya Sakurai

sakurai@cs.tsukuba.ac.jp

1 University of Tsukuba, Tsukuba, Japan

2 RIKEN Center for Computational Science, Kobe, Japan

3 University of Tokyo, Tokyo, Japan
} 
Publisher's Note Springer Nature remains neutral with regard to jurisdictional claims in published maps and institutional affiliations 